\title{
Numerical and experimental study of supersonic combustion chamber at entrance Mach number 4. Part 1: Non-reacting flow
}

\author{
Natalya Fedorova ${ }^{1, *}$ and Marat Goldfeld ${ }^{1}$ \\ ${ }^{1}$ ITAM SD RAS, 4/1 Institutskaya Str., Novosibirsk, 630090, Russia
}

\begin{abstract}
Results of joint experimental and numerical study are presented for a non-reacting 3D flow in a channel with backward-facing steps (BFS) located on top and bottom walls. Experiments were conducted in a hotshort wind tunnel at entrance Mach number 4 , total pressure 9.5 bar and total temperature $2120 \mathrm{~K}$. Argon was injected into the channel from eight round holes, placed on channel walls upstream of the channel expansion. Simulations performed in a 3D RANS approach with $k-\omega$ SST turbulence model give a reasonable agreement with experimental data and allow evaluating the pressure flowfield and argon mass concentration in the channel.
\end{abstract}

\section{Introduction}

The organization of ignition of fuels at a supersonic flow velocity at the inlet to the combustion chamber channel seems to be one of the most difficult and important problems in the scramjet technology development. From technological considerations, the ignition and stabilization of the flame is the first and foremost problem for the scramjet application since ensuring the stabilization of the flame with minimum losses of the total pressure and a high level of heat release are the key to obtaining high engine characteristics [1]. The process of ignition in a supersonic stream depends on many factors, such as the mixture residence time, the local excess fuel ratio, the configuration of the flame stabilizer, the ignition energies, and the physical and kinetic properties of the fuels $[2,3]$.

Despite the fact that ignition and flame stabilization at supersonic flow rates are given great attention, however, the physical and chemical mechanisms, the characteristics of ignition, the processes of flame propagation and heat release in the channel have not been sufficiently studied to reliably predict the processes in the scramjet combustion chamber, especially at high flow rates at the channel inlet.

A decisive role in solving this problem is played by experimental methods. Together with this, the joint use of numerical modeling and experimental methods allows significantly expanding the possibilities of studies of complex supersonic flows in a channel with a mass supply and chemical reactions. In present paper, results of non-reactive

* Corresponding author: nfed@itam.nsc.ru 
flows investigations are presented aimed at experimental and computational study of argon and air mixing in a channel at $\mathrm{M}=4$.

\section{Experimental setup and numerical procedure}

The model of the combustion chamber is a flat channel of $100 \mathrm{~mm}$ wide consisting of an insulator of $200 \mathrm{~mm}$ long, injector and expanding sections, the outline of which is shown in Fig. 1. As a flame stabilizer, the backward facing steps (BFS) of height $h=25 \mathrm{~mm}$, located on the upper and lower walls of the channel were used. The experiments were conducted in a hot-short wind tunnel IT-302 (ITAM SD RAS) with a working time of $80 \div 150 \mathrm{~ms}$. The incoming flow parameters at the entrance of the injector section were as follows: Mach number $\mathrm{M}=4$, the total temperature $\mathrm{T} 0=2120 \mathrm{~K}$, and the total pressure $\mathrm{P} 0=0.95 \mathrm{MPa}$. As the injected gas, at this stage of the investigation, argon was selected, which was fed in the direction of the core of the flow at an angle of 45 degrees from 8 round holes centered at a distance of $8 \mathrm{~mm}$ from the edge of the BFS. The distance from the line of symmetry to the center of the injection hole is $12.5 \mathrm{~mm}$, the distance between the holes is $20 \mathrm{~mm}$, the jet-tofreestream momentum ratio $J=\left(\rho U^{2}\right)_{\mathrm{j}} /\left(\rho U^{2}\right)_{0}$ varies from 2.35 to 8 . In the experiments, the distributions of static pressure and heat fluxes on the model walls, the Pitot pressure at the inlet and outlet of the combustion chamber, and the flow rate of air and injected gas according to the outflow conditions from the closed volume were measured. High-speed shadow visualization was used to determine the flow pattern.

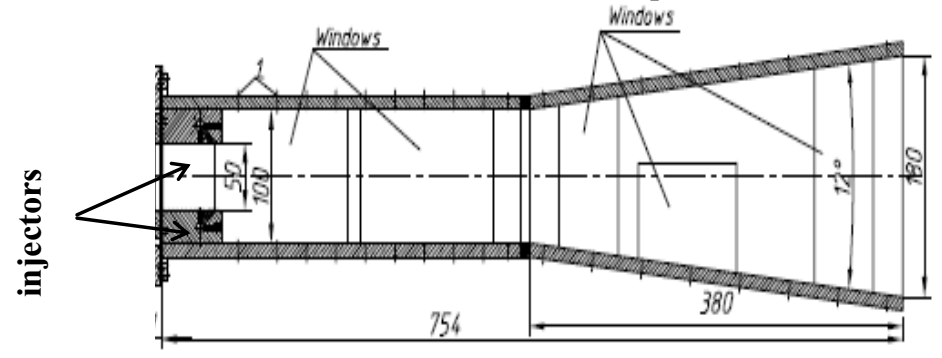

Fig. 1. Outline of injector and expanding sections of experimental model.

Mathematical simulation of the flow was performed under the same inflow conditions based on the Reynolds averaged Navier-Stokes equations supplemented by the $k$ - $\omega$ SST turbulence model using the commercial software ANSYS Fluent 17.0. The results of calculations with the same approach performed earlier to model 2D turbulent supersonic flows in channels, including various gas injections, have shown good agreement with the experimental data $[4,5]$. The present calculations were carried out in 3D taking into account the symmetry of the channel in the vertical and transverse directions. The calculation domain had the same dimensions as the experimental model, and was limited by inlet and outlet sections from the left and right sides, by symmetry planes from the top and front sides and by bottom and back walls. In the computational domain, a structured hexamesh condensed toward the walls was constructed. The final grid contained about 4.5 million cells. Non-dimensional coordinate $\mathrm{y}^{+}$did not exceed 10 at the first cell layer. At the inlet sections, the pressure-inlet type of boundary conditions was applied with the profiles of total and static pressure, temperature and turbulent parameters obtained in the preliminary computation for nozzle and isolator part of the channel. On the walls, the cold wall temperature conditions $\mathrm{Tw}=300 \mathrm{~K}$ were used. 


\section{Results}

As a result of the studies, the structure of the non-reactive flow in the channel was determined, the distribution of pressure and heat fluxes on the model walls, flow parameters as well as argon concentration in the channel were obtained.

Figure 2 shows the static pressure fields in the channel in the symmetry plane $z=0$ for the case without blowing (above) and blowing the argon jet (below). The top picture demonstrates the structure typical for supersonic flows behind BFS. At the inlet to the injector part, the static pressure is $\mathrm{P}_{\mathrm{inf}}=0.85$ bar. After the steps located on the upper and lower walls of the channel, rarefaction waves are formed, in which the pressure is reduced

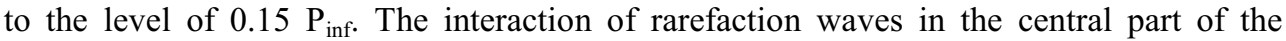
channel reduces the static pressure to the level of $0.06 \mathrm{P}$ inf. Behind the steps, the separation zones are formed with a length of about $2 h$, closing by tail shocks. The shocks meet on the top symmetry line at $x \approx 0.3 \mathrm{~m}$, which leads to a significant pressure increase up to $1.5 \cdot \mathrm{P}_{\text {inf. }}$ Due to reflection from the walls and interference, a symmetrical system of shock waves and rarefaction waves is formed in the channel. The intensity of the shock waves decreases downstream, which is due to the expansion of the channel and the interaction of shock waves with rarefaction waves. For the case with injection, due to presence of the jets pressure maximum increases significantly up to $4 \cdot P_{\text {inf }}$, and the whole wave structure moves upstream. Local static pressure maximum can be observed on the symmetry line in the region of intersection of the shocks generated before the injection holes. By the end of the channel, the pressure level is approximately the same in both cases.
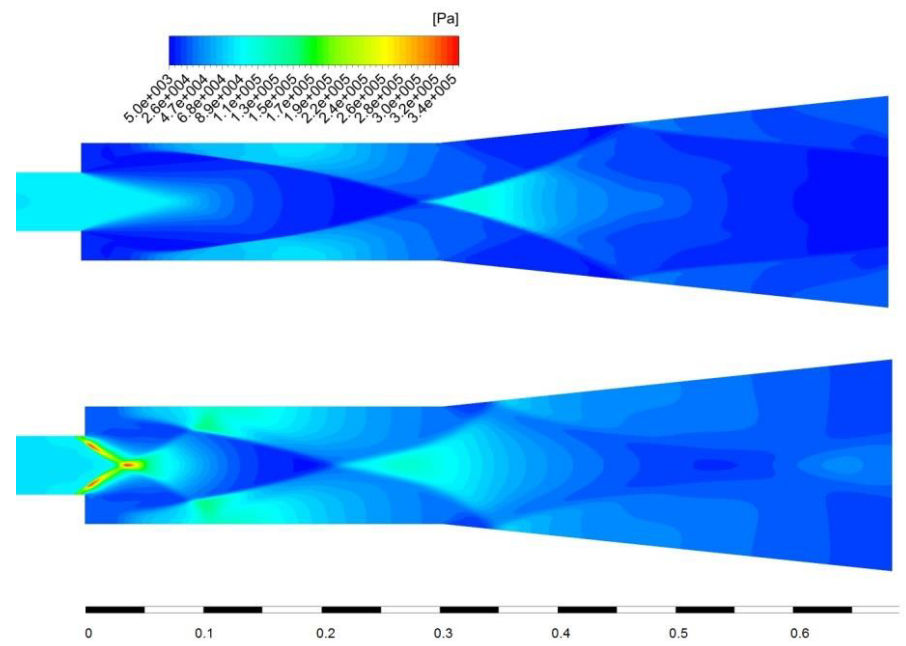

Fig. 2. Static pressure fields in $z=0$ plane for the case without blowing (above) and with blowing the argon jet (below).

In Figure 3, computed and experimental static pressure distributions along the wall at the symmetry plane are compared for the cases without and with argon injection. Despite the fact that injection orifices are shifted from the symmetry plane, a sharp pressure rise before the BFS is observed which is caused by the jet-induced shock. It should be noticed that presence of the jet increases the pressure twice in the separation zone and additional pressure maximum exists at $x=0.14$. Figure shows a satisfactory agreement of numerical and experimental results, nevertheless the pressure maximum for the second case is slightly underpredicted in the computations. 


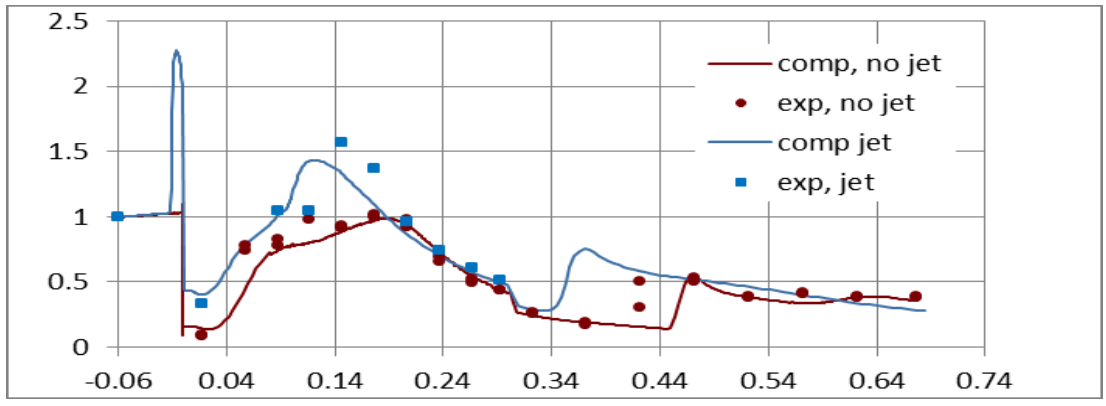

Fig. 3. Experimental and computed static pressure distributions along the wall.

Figure 4 presents the computed argon mass fraction on the bottom wall and in several cross-sections along the channel. The jets do not reach the top symmetry plane, and even at the exit sections, a middle part of the channel does not contain substantial amount of the injected gas. Simultaneously, a rather high argon concentration is observed in the separated region after the BFS. The significant 3D effect in the shape of contours and mean values of argon concentration far downstream from the injection could be seen due to the back wall presence.

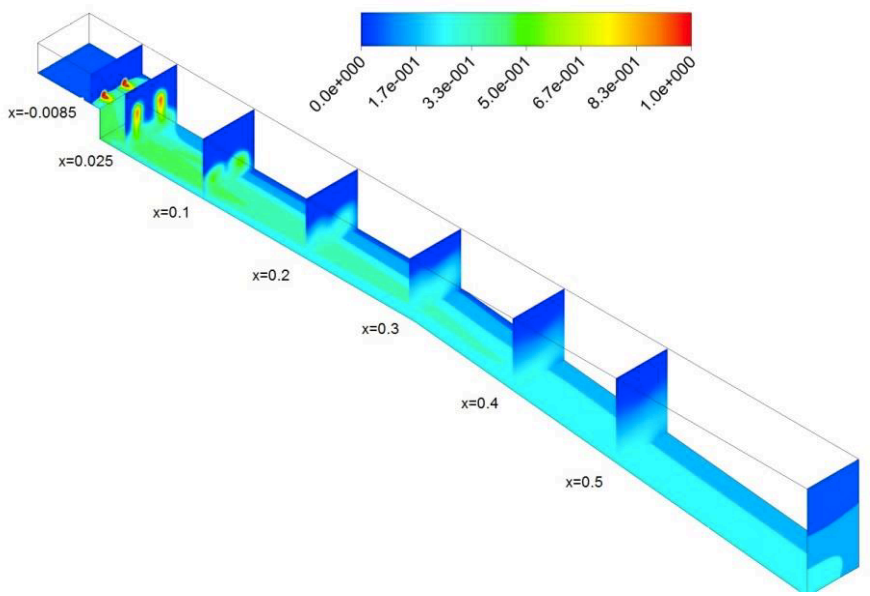

Fig. 4. Computed argon mass concentration at various $x$-cross-sections.

The work was supported by the Russian Foundation for Basic Research grant 17-08-01158 A.

\section{References}

1. E.T. Curran, J. Propuls. Power 17, 6 (2001)

2. C.C. Rasmussen, J.F. Driscoll, K.Y. Hsu, J.M. Donbar, M.R. Gruber, C.D. Carter, J. Propul. Power 21 (2005)

3. D.J. Micka, J.F. Driscoll, Combust. Flame 159, 1205 (2012)

4. M.A. Goldfeld, Yu.V. Zakharova, N.N. Fedorova, Thermophys. Aeromech. 19, 4 (2012)

5. N.N. Fedorova, I.A. Fedorchenko, A.V. Fedorov J. Appl. Mech. Tech. Phys. 54, 2 (2013) 